\title{
Segmentation of Noisy and Textured Images by an Expectation Maximization and Gradient Descent Iteration Method
}

\author{
Zhenzhou WANG ${ }^{1, a}$, Yongming YANG ${ }^{2, b}$ \\ ${ }^{1,2}$ Shenyang Institute of Automation, Chinese Academy of SciencesShenyang City, PR China \\ aemail: wangzhenzhou@sia.cn, bemail: yangyongming@sia.cn
}

Keywords: Image Segmentation; Expectation Maximization; Gibbs Distribtion

\begin{abstract}
Segmentation of noisy and textured images remains challenging in both accuracy and computation efficiency.In this paper, we propose a new method for segmentation of noisy and textured images. The proposed method is based on the famous Expectation Maximization (EM) methodwhich calculates the global parameters of the image and Gibbs distribution which calculates the local parameters of the image.With the global parameters of the objects and the background computed from EM, a pre-segmentation is achieved. Then we propose a gradient descent iteration (GDI) method to achieve the final segmentation by minimizing the sum of local energy.Experimental results show that the proposed method is more effective than the state of art Normalized Cut method in segmenting noisy and textured images.
\end{abstract}

\section{Introduction}

Image segmentation plays an important role in computer vision problems. Due to the variety and complexity of the images captured in the real world, robust segmentation of the objects from the background remains a bottleneck problem for many types of images, especially the noisy and textured images. Much of the past research work has been directed to stochastic modeling methods[1]-[14]. Usually, the stochastic modeling methods assume the image as a Markov Random Field (MRF) which complies with Gibbs distribution. The Gibbs distribution characterizes the interaction of the neighboring pixels. Thus, it belongs to the local property of the image. Markov Chain Monte Carlo (MCMC)[2][15]is a popular way to estimate the parameters of the Gibbs distribution. Unfortunately, it does not converge to correct labeling in most cases for the segmentation.Thus, its application to segmentation problems is very limited. In addition, its effectiveness in estimating the Gibbs parameteris only limited to some specific textured images. In contrast to the local property, stochastic modeling methodsassume different pixel classes complying with Gaussian distribution, which represents the global property of the image. Usually, the expectation maximization (EM) algorithm[3]is used to estimate the means and variances of different pixel classes and its effectiveness has been widely accepted.However, the computation complexity of EMis not satisfying, which limits its applications greatly.

As the most representative stochastic modeling method, Maximum A Posteriori (MAP)combines the local property and global property of the image and tries to find an overall optimal estimation of the Gibbs parameters and the Gaussian parameters. However, its computation complexity becomes intractable with the increase of image resolution and the number of the pixel classes.To deal with the intractability of computation complexity, different methods have been proposed, e.g. dynamic programming [9],Iterated Conditional Modes (ICM) [11], annealing method [12] and maximization of local energy and global energy independently [1]. Unfortunately, none of these methods have achieved satisfying accuracy and computation complexity in segmenting noisy and textured images.

An alternative research direction is to use graph cuts method[16][17] to segment noisy and textured images. However, their poor performance does not attract much attention in segmenting noisy textured images. Many researchers[13][14] have combined MAP and graph cuts to segment noisy images. Since they just use graph cuts to find the solutions of MAP, their methods can be classified as MAP method. 
In this paper, we propose anExpectation Maximization and Gradient Descent Iteration (EM-GDI) method to segment noisy and textured images. The proposedmethodcomputes the Gaussian means from the famous EM method. A pre-segmentation is then acquired from the computed Gaussian means of different pixel classes. Due to the noise and isolated textures, we propose a gradient descent iteration (GDI) method to minimize the local energy of the pre-segmented image. Experimental results show that the proposed method is more effective in segmenting noisy and textured images than the state of art Normalized Cut method.

This paper is organized as follows. Section 2 analyzes the image property in the sense of global and local on which the proposed method is based on. Experimental results are given in Section 3 to verify the proposed method. In Section 4 conclusion is drawn and future work is discussed.

\section{Proposed Method}

\section{Image Property Analysis}

The property of the image includes: 1) the local property which is characterized by Gibbs distribution; 2) the global property which is characterized by Gaussian distribution.

The Gibbs distribution characterizes the image as a Markov random field $\mathrm{X}=\left\{X_{\mathrm{s}}\right\}$ which has a joint distribution.

$$
\mathrm{P}(\mathrm{X}=\mathrm{x})=\frac{1}{\mathrm{Z}} \mathrm{e}^{-\mathrm{U}(\mathrm{x})}
$$

$$
\begin{aligned}
& \text { Where } \\
& \mathrm{U}(\mathrm{x})=\sum_{\mathrm{c} \in \mathrm{C}} \mathrm{Vc}(\mathrm{x}) \\
& \mathrm{Z}=\sum_{\mathrm{x} \in \mathrm{L}} \mathrm{e}^{-\mathrm{U}(\mathrm{x})}
\end{aligned}
$$

Where $U(x)$ is the energy function and $\operatorname{Vc}(\mathrm{x})$ is the potential function associated with clique c. $\mathrm{Z}$ is the normalizing partition function which is defined over the whole lattice $\mathrm{L}$. The potential function $\mathrm{Vc}(\mathrm{x})$ for a non-single-site clique cis defined as follows.

$$
\operatorname{Vc}(\mathrm{x})=\left\{\begin{array}{cc}
-\beta ; & \text { all values are equal } \\
\beta ; & \text { else }
\end{array}\right.
$$

The parameter set $\theta \mathrm{x}$ which characterizesthe neighborhood system can be written in the following form, $\theta x=\left\{\beta_{1}, \beta_{2}, \beta_{3}, \beta_{4}, \beta_{5}, \beta_{6}, \beta_{7}, \beta_{8}\right\}$. MCMC can be used to estimate the parameter set $\theta x$ for some specific textures.

The Gaussian distribution characterizes the image as the random variables $\left\{\mathrm{Y}_{\mathrm{n}}, 0<\mathrm{n}<M N+1\right\}$ with the class labels $\left\{\mathrm{X}_{\mathrm{n}}, 0<\mathrm{n}<M N+1\right\}$. Each label takes on $\mathrm{lx}$ possible values and denoted as $\mathrm{X}_{\mathrm{n}} \in$ $\left\{0, \ldots, l_{x}-1\right\}$. The conditional distribution of $Y_{n}$ given $X_{n}$ is formulated in the Gaussian format.

$$
\mathrm{P}\left(\mathrm{y}_{\mathrm{n}} \mid \mathrm{x}_{\mathrm{n}}\right)=\frac{1}{\sqrt{2 \pi \sigma_{\mathrm{x}_{n}}^{2}}} \mathrm{e}^{-\frac{1}{2 \sigma_{\mathrm{x}_{n}}^{2}}\left(\mathrm{y}_{\mathrm{n}}-\mu_{\mathrm{x}_{\mathrm{n}}}\right)^{2}}
$$

Where $\mu_{\mathrm{x}_{\mathrm{n}}}$ and $\sigma_{\mathrm{x}_{\mathrm{n}}}^{2}$ are the mean and variance of the class label $\mathrm{x}_{\mathrm{n}}$. The marginal Gaussian distribution of $\mathrm{y}_{\mathrm{n}}$ is given by the following equation.

$$
\mathrm{P}\left(\mathrm{y}_{\mathrm{n}}\right)=\sum_{\mathrm{m}=0}^{\mathrm{lx}-1} \frac{\pi_{\mathrm{m}}}{\sqrt{2 \pi \sigma_{\mathrm{m}}^{2}}} \mathrm{e}^{-\frac{1}{2 \sigma_{\mathrm{m}}^{2}}\left(\mathrm{y}_{\mathrm{n}}-\mu_{\mathrm{m}}\right)^{2}}
$$

Where $\pi_{\mathrm{m}}$ is the probability thatthe class label takes on the value $m$. The distribution of the entire observed image sequence $\left\{\mathrm{Y}_{\mathrm{n}}, 0<\mathrm{n}<M N+1\right\}$ is formulated as follows.

$$
\mathrm{P}(\mathrm{y})=\prod_{\mathrm{n}=1}^{\mathrm{MN}} \sum_{\mathrm{m}=0}^{\mathrm{lx}-1} \frac{\pi_{\mathrm{m}}}{\sqrt{2 \pi \sigma_{\mathrm{m}}^{2}}} \mathrm{e}^{-\frac{1}{2 \sigma_{\mathrm{m}}^{2}}\left(\mathrm{y}_{\mathrm{n}}-\mu_{\mathrm{m}}\right)^{2}}
$$

EM algorithm is usually used to find the parameter set $\theta y=\left\{\mu_{0}, \sigma_{0}^{2}, \ldots, \mu_{\mathrm{lx}-1}, \sigma_{\mathrm{lx}-1}^{2}\right\}$ which maximize the probability $\mathrm{P}(\mathrm{y})$. 
The MAP segmentation combines the local property (Eq.1) and global property (Eq.7) of the image and is characterized by the posteriori distribution.

$$
\mathrm{P}(\mathrm{X}=\mathrm{x} \mid \mathrm{Y}=\mathrm{y})=\frac{\mathrm{P}(\mathrm{Y}=\mathrm{y} \mid \mathrm{X}=\mathrm{x}) \mathrm{P}(\mathrm{X}=\mathrm{x})}{\mathrm{P}(\mathrm{Y}=\mathrm{y})}
$$

For the computation simplicity, its logarithm format is usually used. Combining Eq. 1, 2, 7 and 8, we get the MAP formulation with joint local property and global property:

$$
\ln \mathrm{P}(\mathrm{X}=\mathrm{x}, \mathrm{Y}=\mathrm{y})=-\ln \mathrm{Z}-\sum_{\mathrm{c} \in \mathrm{C}} \mathrm{Vc}(\mathrm{x})+\ln \left(\prod_{\mathrm{n}=1}^{\mathrm{MN}} \sum_{\mathrm{m}=0}^{\mathrm{lx}-1} \frac{\pi_{\mathrm{m}}}{\sqrt{2 \pi \sigma_{\mathrm{m}}^{2}}} \mathrm{e}^{-\frac{1}{2 \sigma_{\mathrm{m}}^{2}}\left(\mathrm{y}_{\mathrm{n}}-\mu_{\mathrm{m}}\right)^{2}}\right)
$$

From the above equation, it is seen that it is intractable to maximize the parameter $\theta \mathrm{x}$ and $\theta \mathrm{y}$ at the same time. Hence, in [1], a pseudo MAP method is proposed to maximize the Gibbs distribution with MCMC estimation (the first two terms in Eq. 10) and Gaussian distribution with EM estimation (the third term in Eq. 10) independently. However, MCMC fails frequently in estimating the parameter set correctly for many images (both textured and non-textured). For instance, it randomly estimated $\bar{\theta} \mathrm{x}$ as $\{0.84,0.80,0.75,0.70,0.64,0.46,0.53,0.73\}$ for the synthesized image with $\theta \mathrm{x}=$ $\{1,1,1,1,1,1,1,1\}$; It randomly estimated $\bar{\theta} \mathrm{x}$ as $\{0.18,1.13,0.55,1.57,-0.25,-0.61,-0.79,-0.94\}$ for the synthesized image with $\theta x=\{1,2,3,4,-1,-2,-3,-4\}$. In [1], an iteration method based on the estimated parameter set $\bar{\theta} \mathrm{x}$ is used to converge the pre-segmentation result by EM. The work conducted in this search makes it clearer that parameter set $\bar{\theta} x$ is superfluous for the segmentation. Thus, we abandon it in this research and modify the method in [1] as a gradient descent iteration (GDI) method [18] which iterates according to the following equation until it converges:

$$
\overline{\mathrm{I}_{\mathrm{l}+1}}(\mathrm{u}, \mathrm{v})=\frac{1}{9} \sum_{\mathrm{i}=-1}^{1} \sum_{\mathrm{j}=-1}^{1} \overline{\mathrm{I}_{\mathrm{l}}}(\mathrm{u}+\mathrm{i}, \mathrm{v}+\mathrm{j})(10
$$

Where $\bar{I}_{1}(u, v)=I(u, v)$ denotes the pixel value at position $(u, v)$ in the original image. $l$ denotes the least iteration number that Eq. 10 converges.

\section{Experimental Results}

First, we synthesized a image with $\theta y=\{50,30,100,30,150,30\}$ and it is shown in Fig. 1 (a). The computed mean values by EM are 54.1663, 119.3745 and 151.6683 respectively.Fig. 1 (b) shows the pre-segmentation result by EM and (c) shows the final segmentation result by GDI.We also show the segmentation result by Normalized Cut in (d) for comparison. As can be seen, the proposed method achieves a better result.

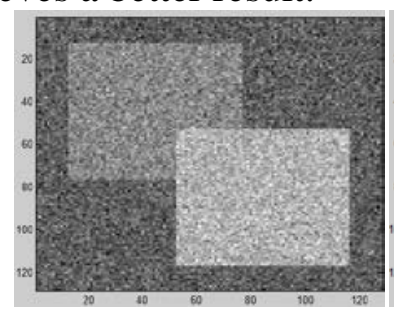

(a)

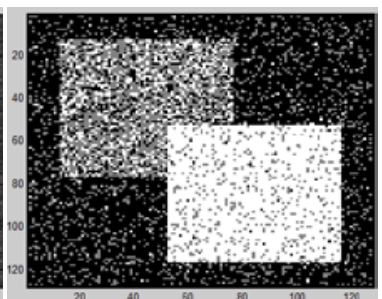

(b)

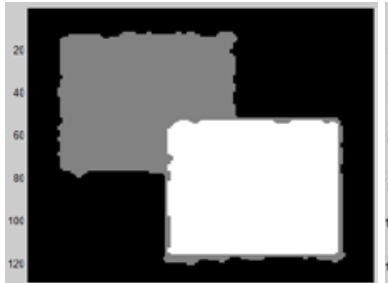

(c)

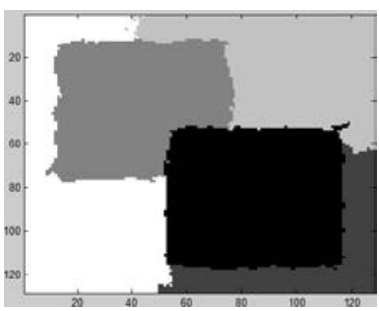

(d)

Fig. 1 Comparison of the proposed methodwith Normalized Cut (a) Synthesized image with $\theta y=\{50,30,100,30,150,30\}$; (b) Pre-segmentation by EM; (c) Final segmentation by GDI method;

(d) Segmentation result by Normalized Cut

We synthesized another image with $\theta y=\{50,50,100,50,150,50\}$ as shown in Fig. 2 (a) and the computed mean values by EM are 41.8904, 76.3151 and 130.4943 respectively. Fig. 2 (b) shows the pre-segmentation result by EM and (c) shows the final segmentation result by GDI. (d) shows the Normalized Cut result for comparison. As can be seen, when the noise increases, the proposed method can still achieves an acceptable result while Normalized Cut fails. 


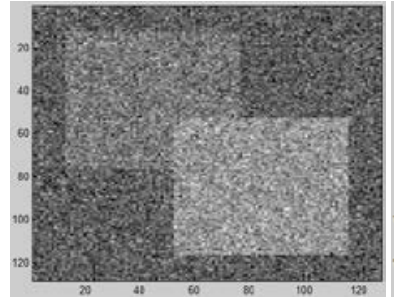

(a)

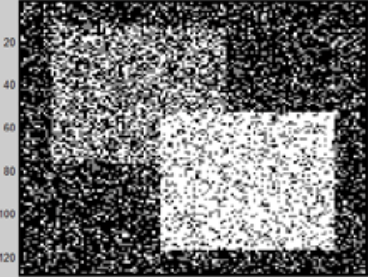

(b)

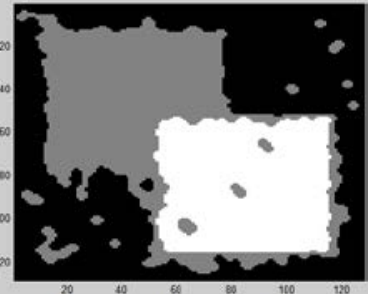

(c)

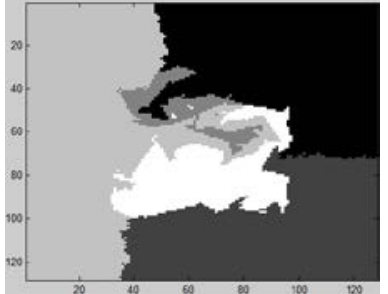

(d)

Fig. 2 Comparison of the proposed method with Normalized Cut (a) Synthesized image with $\theta y=\{50,50,100,50,150,50\}$; (b) Pre-segmentation by EM; (c) Final segmentation by GDI method;

(d) Segmentation result by Normalized Cut

Then, we synthesized a textured image with parameter $\theta x=\{0,0,0,0,3,-3,-3,3\}$ as shown in Figs. 3-6 (a). We add different noise varying as [30, 20, 10, 5] to these synthesized images. From the segmentation results, it is seen that the proposed method is superior to Normalized Cut in segmenting textured images.

As last, we use two real images to compare the performances of the proposed method over Normalized Cut method as shown in Fig. 7 and Fig. 8. As can be seen, the proposed method is better.

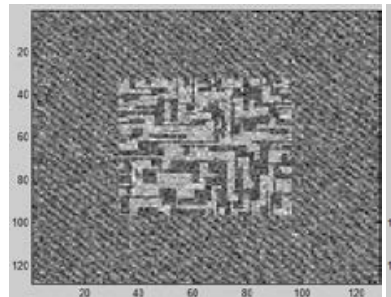

(a)

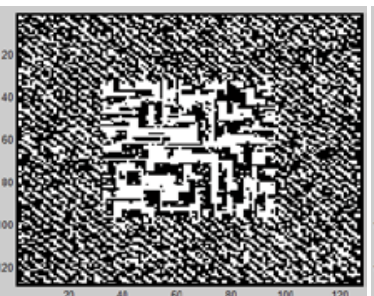

(b)

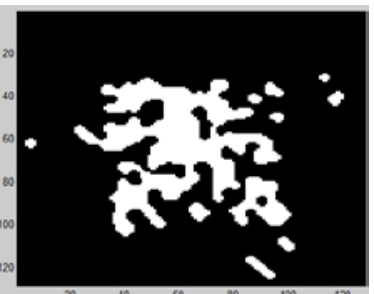

(c)

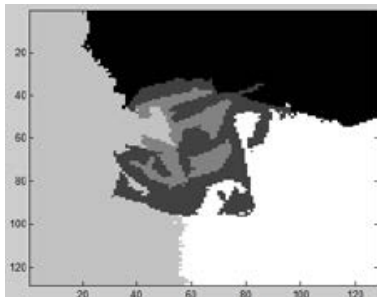

(d)

Fig. 3 Comparison of the proposed method with Normalized Cut (a) Synthesized image with $\theta y=\{50,30,100,30\}$; (b) Pre-segmentation by EM; (c) Final segmentation by GDI method; (d) Segmentation result by Normalized Cut

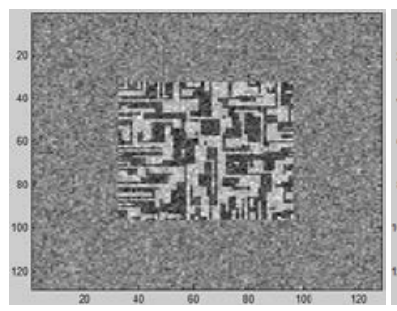

(a)

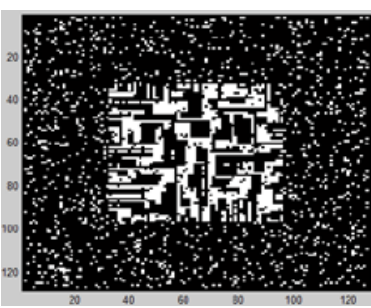

(b)

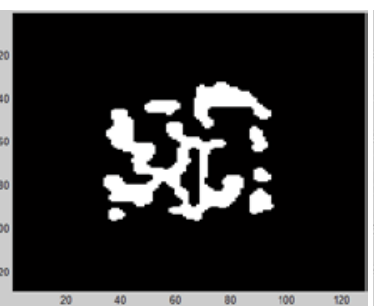

(c)

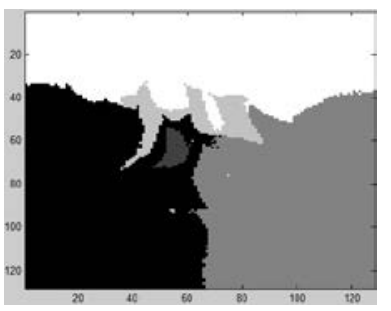

(d)

Fig. 4 Comparison of the proposed method with Normalized Cut (a) Synthesized image with $\theta y=\{50,20,100,20\}$; (b) Pre-segmentation by EM; (c) Final segmentation by GDI method; (d) Segmentation result by Normalized Cut

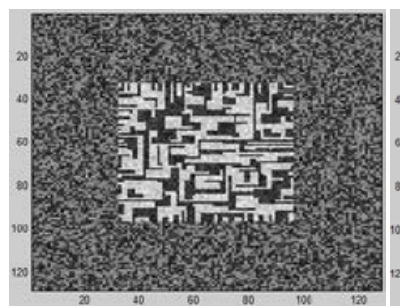

(a)

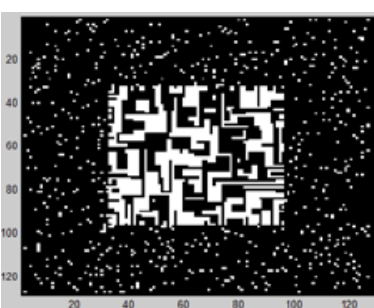

(b)

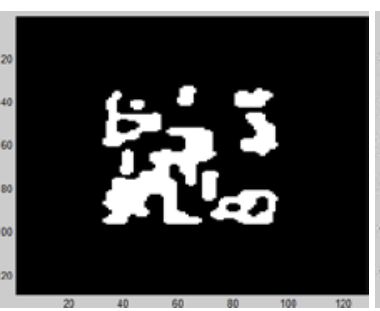

(c)

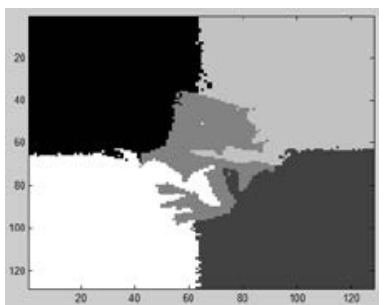

(d)

Fig. 5 Comparison of the proposed method with Normalized Cut (a) Synthesized image with $\theta y=\{50,10,100,10\}$; (b) Pre-segmentation by EM; (c) Final segmentation by GDI method; (d) Segmentation result by Normalized Cut 


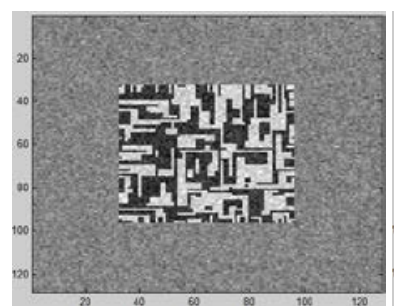

(a)

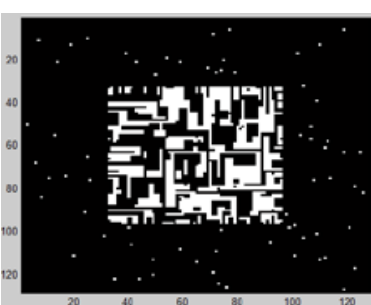

(b)

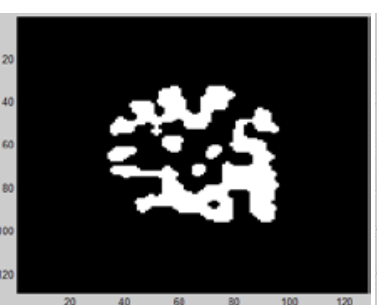

(c)

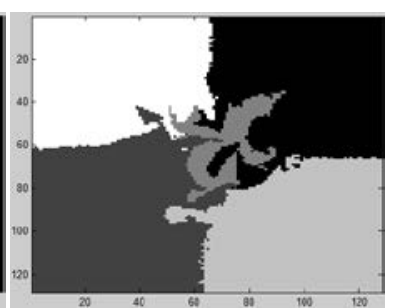

(d)

Fig. 6 Comparison of the proposed method with Normalized Cut (a) Synthesized image with $\theta y=\{50,5,100,5\}$; (b) Pre-segmentation by EM; (c) Final segmentation by GDI method; (d)

Segmentation result by Normalized Cut

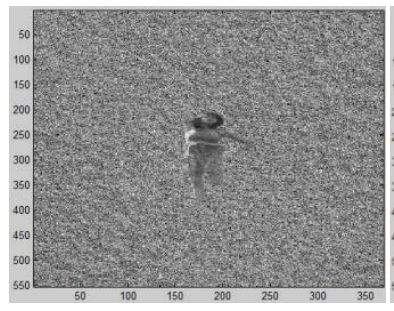

(a)

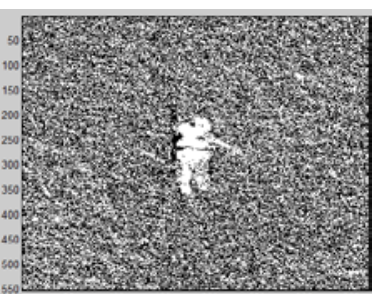

(b)

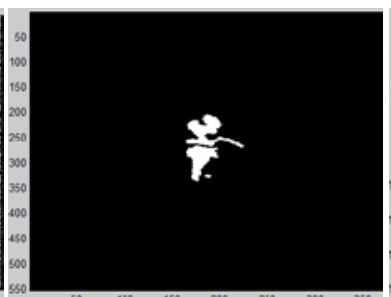

(c)

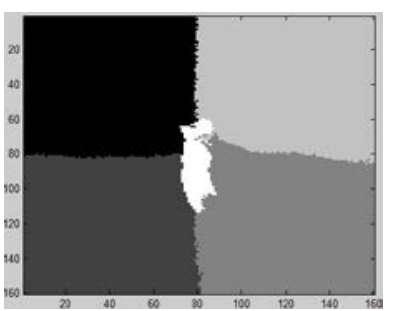

(d)

Fig. 7 Comparison of the proposed method with Normalized Cut (a) A noisy image; (b) Pre-segmentation by EM; (c) Final segmentation by GDI method; (d) Segmentation result by Normalized Cut

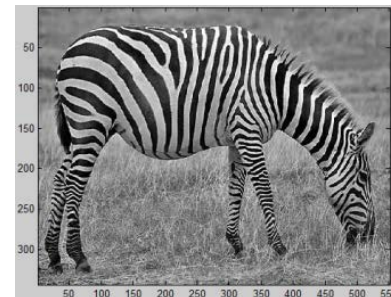

(a)

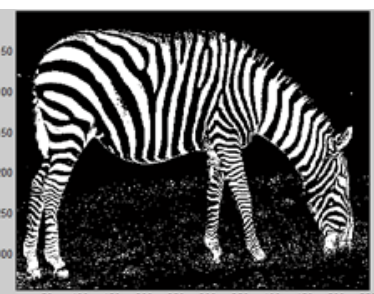

(b)

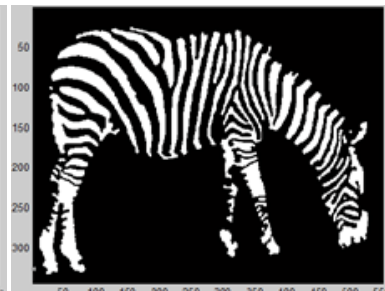

(c)

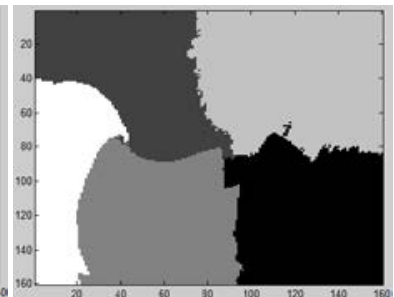

(d)

Fig. 8 Comparison of the proposed method with Normalized Cut (a) A textured image; (b) Pre-segmentation by EM; (c) Final segmentation by GDI method; (d) Segmentation result by Normalized Cut

\section{Conclusion and Future Work}

In this paper, anexpectation maximization and gradient descent iteration (EM-GDI)method is proposed to segment the noisy and textured images based on the global and local properties of the image. Experimental results verified that the proposed method is superior to the state of art Normalized Cut method in segmenting noisy and textured images.

The future work includes, but not limited to: (1), coming up with more efficient and robust methods than EM to calculate the global parameters of the image; (2), explore the local property of the image more thoroughly.

\section{References}

[1] Z.Z. Wang and Y.M. Zhang, " Segmentation of fuzzy images: a novel and fast two-steppseudo MAPmethod," Machine Vision and Applications. Vol. 23, pp.1209-1218, 2012.

[2] C.S. Won and R.M. Gray, "Stochastic Image Processing," Information technology: Transmission, Processing, and Storage, 2004.

[3] A.P. Dempster, N.M. Laird and D.B. Rubin, "Maximum Likelihood from Incomplete Data via the EM Algorithm,"Journal of the Royal Statistical Society, Series B, Vol. 39, No. 1 pp. 1-38, 1977. 
[4] B. Braathen, W. Pieczynski, and P. Masson, "Global and Local Methods of Unsupervised Bayesian Segmentation of Images," Machine Graphics Vision 2, No. 1, 39-52, 1993.

[5]H. Caillol, A. Hillon, and W. Pieczynski, "Fuzzy Random Fields and Unsupervised Image Segmentation," IEEE Trans. Geoscience Remote Sensing GE-31, No. 4, 801-810, 1993.

[6] H. Caillol, W. Pieczynski, and A. Hillon, "Estimation of Fuzzy Gaussian Mixture and Unsupervised Statistical Image Segmentation," IEEE Trans. Image Process IP-6, No. 3, 425-440, 1997.

[7] P. Masson and W. Pieczynski, "SEM Algorithm and Unsupervised Statistical Segmentation of Satellite Images," IEEE Trans. Geoscience Remote Sensing GE-34, No. 3, 618-633, 1993.

[8] H. Derin, H. Elliott, and J. Kuang, "A New Approach to Parameter Estimation for Gibbs Random Fields," in Proc. IEEE 1985 Conf. ASSP, Tampa, FL, Mar. 1985.

[9] H. Derin and H. Elliott, " Modeling and Segmentation of Noisy and Textured Images Using Gibbs Random Fields," . IEEE Trans. Pattern Analysis Machine Intelligence, 9 (1), 39-55. 1987.

[10] H. Derin and W. S. Cole, "Segmentation of Textured Images Using Gibbs Random Fields," Computer Vision, Graphics, Image Processing, vol. 35, pp. 72-98, 1986.

[11] J. Besag, "On the Statistical Analysis of Dirty Pictures, " J. Roy. Statist. Soc. B, vol. 48, No. 3, pp. 259-302, 1986.

[12] S. Geman and D. Geman, "Stochastic Relaxation, Gibbs Distributions, and the Bayesian Restoration of Images," IEEE Transaction on Pattern Anal Machine Intell vol. PAMI-6, pp. 721-741, Nov. 1984.

[13] D.M. Greig, B.T. Porteous and A.H. Seheult, "Exact Maximum A Posteriori Estimation for Binary Images," Journal of the Royal Statistical Society. Series B. Vol. 51, No. 2, pp.271-279, 1989.

[14] S.F. Chen, L.L. Cao, Y.M. Wang, J.Z. Liu and X.O. Tang,"Image Segmentation by MAP-ML Estimations", IEEE Transactions on Image Processing, Vol. 19, No. 9, Sept. 2010.

[15] L.Wang, J. Liu, S.Z. Li, "MRF Parameter Estimation by MCMC Method," Pattern Recognition 33, 1919-1925. 2000.

[16] J. Shi and J. Malik, "Normalized Cuts and Image Segmentation," IEEE Trans. on Pat. Analysis and Machine Intelligence, Vol. 22, No. 8, August, 2000.

[17] Y. Boykov and G. Funka-lea, "Graph Cuts and Efficient N-D Image Segmentation," International Journal of Computer Vision, Vol. 70 (2), pp. 109-131, 2006.

[18] Z.Z. Wang and G.L. Li, "A New Cross Based Gradient Descent Search Algorithm for Block Matching in MPEG-4 Encoder," Chinese Journal of Electronics, Vol.12, No.4, pp. 648-651,Oct. 2003. 